\title{
Scattering matrices with finite phase shift and the inverse scattering problem
}

\author{
Pavel Kurasov \\ Alexander von Humboldt fellow, Department of Mathematics, Ruhr-University Bochum, \\ 44780 Bochum, Germany \\ Department of Mathematical and Computational Physics, St Petersburg University, 198904 \\ St Petersburg, Russia \\ Department of Mathematics, Luleå University, 97187 Luleå, Sweden \\ Received 3 July 1995, in final form 19 February 1996
}

\begin{abstract}
The inverse scattering problem for the Schrödinger operator on the half-axis is studied. It is shown that this problem can be solved for the scattering matrices with arbitrary finite phase shift on the real axis if one admits potentials with long-range oscillating tails at infinity. The solution of the problem is constructed with the help of the Gelfand-Levitan-Marchenko procedure. The inverse problem has no unique solution for the standard set of scattering data which includes the scattering matrix, energies of the bound states and corresponding normalizing constants. This fact is related to zeros of the spectral density on the real axis. It is proven that the inverse problem has a unique solution in the defined class of potentials if the zeros of the spectral density are added to the set of scattering data.
\end{abstract}

\section{Introduction}

The inverse scattering problem for the Schrödinger operator

$$
H u=-\frac{\mathrm{d}^{2} u}{\mathrm{~d} x^{2}}+V(x) u
$$

on the half-axis with the Dirichlet boundary condition at the origin $u(0)=0$ is investigated in the present paper. This problem has been studied for real potentials from the Faddeev class, i.e. with a finite first momentum

$$
\int_{0}^{\infty}(1+x)|V(x)| \mathrm{d} x<\infty .
$$

One-to-one correspondence between the scattering data and potentials was established. The set of scattering data included the scattering matrix, the energies of the bound states and corresponding normalizing constants. See [9] for an excellent review of the modern results in this direction. This problem is closely related to the inverse spectral problem [11], which was originally studied by Gelfand, Levitan and Marchenko $[12,18]$ and recently by Gesztesy and Simon [13]. The relations with the inverse spectral problem are usually used to prove the uniqueness results for the potential. Thus the existence problem appears to be very important during the investigation of the inverse scattering problem. The existence of the potential has been proven only for the scattering matrices $S(k)$ with a negative phase shift on the real axis

$$
\left.\arg S(k)\right|_{-\infty} ^{\infty} \leqslant 0
$$


because the phase shift on the real axis for potentials from the Faddeev class is related to the number of bound states through Levinson's theorem. The main goal of the present paper is to show that the inverse problem can be solved for scattering matrices with any finite phase shift on the real line (not only negative ones). The set of potentials has to be extended in order to include potentials with long-range oscillating tails at infinity. Such potentials with asymptotics in the form $V(x) \sim_{x \rightarrow \infty} c \sin 2(b x+\beta) /(x+1)$ were considered in connection with the bound states embedded in a continuous spectrum [1,10,19,27]. We confine our consideration to the case of negative bound states. Positive- and zero-energy eigenvalues give no contribution to the scattering matrix and can be considered separately. The class of admissible potentials includes potentials decreasing at infinity such as $1 /|x+1|^{2}$. The corresponding Schrödinger operators can have an infinite number of bound states, but our consideration is confined to the case when the number of bound states is finite.

It has been shown that the standard set of scattering data (scattering matrix, eigenvalues and corresponding constants) does not provide enough information to reconstruct a unique potential with long-range oscillations $[14,15]$. This phenomenon is related to the fact that the spectral density can have zeros on the positive part of the real axis. The spectral density $\rho(k)$ for the Schrödinger operator on the half-axis is defined by the Jost function $F(k)$ : $\rho(k)=(2 / \pi)\left(1 /\left(|F(k)|^{2}\right)\right.$. The Jost functions for the potentials from the Faddeev class are bounded in the closed upper half-plane and the spectral density does not vanish on the positive part of the real line. This is not true for potentials which do not belong to the Faddeev class. For example the potentials

$$
\begin{aligned}
& V_{b}(x)=16 b^{2} \frac{1-(b x+B) \sin 2(b x+\delta)-\cos 2(b x+\delta)}{(2 b x+2 B-\sin 2(b x+\delta))^{2}} \\
& B=\frac{b(a+\bar{a})\left(a \bar{a}+b^{2}\right)}{\left(a^{2}+b^{2}\right)\left(\bar{a}^{2}+b^{2}\right)} \quad \mathrm{e}^{2 \mathrm{i} \delta}=\frac{b-\mathrm{i} a}{b+\mathrm{i} a} \frac{b-\mathrm{i} \bar{a}}{b+\mathrm{i} \bar{a}} \quad \text { Re } a>0
\end{aligned}
$$

correspond to one and the same scattering matrix $S(k)=(k-\mathrm{i} a)(k-\mathrm{i} \bar{a}) /(k+$ $\mathrm{i} a)(k+\mathrm{i} \bar{a})$. The Jost function has singularities at the points $k= \pm b: F(k)=$ $(k+\mathrm{i} a)(k+\mathrm{i} \bar{a}) /(k-b)(k+b)$.

Similar examples of potentials having the same scattering data on the entire axis were constructed in $[2,3,7,24]$. All these examples are related to the special behaviour of the scattering matrix at the origin. The family of potentials $V_{b}(x)$ has nonstandard behaviour for the spectral density $\rho(k)$ outside the origin: it vanishes at $k= \pm b$. In order to have a unique solution for the inverse problem, the positions of the zeros of the spectral density have to be added to the set of scattering data. An arbitrary finite number of such zeros is considered in the present paper. The corresponding scattering matrix can have a positive phase shift on the real axis. We show that the inverse scattering problem on the half-axis can be solved for scattering matrices with an arbitrary finite phase shift on the real axis. The generalized Levinson theorem relates the phase shift to the number of bound states and zeros of the spectral density.

The paper is organized as follows. First the sets of admissible scattering matrices and Jost functions are described. Then the generalized set of scattering data is introduced. Next the set of potentials with long-range oscillating tails at infinity is defined. It is shown that solution of the inverse problem for the defined class of scattering data can be found in the described class of potentials. Uniqueness of the potential is finally established using the relations with the inverse spectral problem. 


\section{Scattering matrices and Jost functions}

We are going to solve the inverse scattering problem on the half-axis for the scattering matrices from the following set $\mathcal{S}$ :

Definition 1. The set $\mathcal{S}$ of scattering matrices consists of all continuous functions on the real line with the following properties:

(1) $|S(k)|=1$;

(2) $S(\infty)=1$;

(3) $S(-k)=\overline{S(k)}$;

(4) $S(k)-1=\int_{-\infty}^{\infty} \sigma(t) \mathrm{e}^{\mathrm{i} k t} \mathrm{~d} t, \int_{-\infty}^{\infty}|\sigma(t)| \mathrm{d} t<\infty$.

We note, that the subset of scattering matrices corresponding to potentials with a finite first momentum can be selected by an additional condition (equation (1)), which follows from Levinson's theorem. This subset of the scattering matrices will be denoted by $\mathcal{S}_{F}$. The solution of the inverse problem for such scattering matrices can be derived using the properties of the Jost function $F(k)$. One can prove that the Jost functions for the potentials with a finite first momentum possess the following properties:

(1) analytical in the open upper half-plane $\operatorname{Im} k>0$;

(2) continuous in the closed upper half-plane $\operatorname{Im} k \geqslant 0$;

(3) $\lim _{k \rightarrow \infty} F(k)=1$;

(4) $F(k)=\overline{F(-\bar{k})}$;

(5) possess the following representation:

$$
F(k)=1+\int_{0}^{\infty} f(t) \mathrm{e}^{\mathrm{i} k t} \mathrm{~d} t \quad \int_{0}^{\infty}|f(t)| \mathrm{d} t<\infty .
$$

All functions satisfying properties (1)-(5) form Faddeev's class of Jost functions and will be denoted by $\mathcal{F}_{F}$ hereafter. Levinson's theorem relates the phase shift on the real axis to the number of bound states $N_{b s}$ of the corresponding Schrödinger operator:

$$
\begin{aligned}
& \left.\arg S(k)\right|_{-\infty} ^{\infty}=-4 N_{b s} \pi \quad \text { if } F(0) \neq 0 \\
& \left.\arg S(k)\right|_{-\infty} ^{\infty}=-\left(2 N_{b s}+1\right) 2 \pi \quad \text { if } F(0)=0 .
\end{aligned}
$$

In order to solve the inverse problem for the scattering matrices $S(k)$ from $\mathcal{S}$ the set of admissible Jost functions has to be extended. The phase shift of $S(k)$ on the real axis is always finite. If it is nonpositive, then $S(k) \in \mathcal{S}_{F}$ and the inverse problem can be solved in the class of potentials with a finite first momentum. One can find function $F(k) \in \mathcal{F}_{F}$ such that $S(k)=F(-k) / F(k)$. The potential corresponding to this Jost function can be calculated with the help of the Gelfand-Levitan-Marchenko procedure. If the phase shift is positive, then the scattering matrix can be presented in the following form:

$$
S(k)=\frac{k-\mathrm{i} a_{0}}{k+\mathrm{i} a_{0}}\left(\prod_{j=1}^{N_{\text {sing }}} \frac{k-\mathrm{i} a_{j}}{k+\mathrm{i} a_{j}} \frac{k-\mathrm{i} \bar{a}_{j}}{k+\mathrm{i} \bar{a}_{j}}\right) S_{F}(k)=S_{\text {sing }}(k) S_{F}(k)
$$

where $S_{F}(k) \in \mathcal{S}_{F}$. Constants $a_{0}>0, \operatorname{Re} a_{j}>0$ can be chosen arbitrarily. It is impossible to find $F(k) \in \mathcal{F}_{F}$ such that $S_{\text {sing }}(k)=F(-k) / F(k)$ holds. The Jost function corresponding to $S_{\text {sing }}(k)$ can be found only with singularities on the real line. Thus we are going to consider the Jost functions to be continuous in the closed upper half-plane $\operatorname{Im} k \geqslant 0$ except at a finite number of points $\pm b_{j}, b_{j}>0, j=1,2, \ldots, N_{\text {sing }}$ and may be at the origin. The function can have simple poles at these points. We are going to speak about the zero-energy singularity in the case when the Jost function has a pole at the origin. If the Jost function is equal to zero at the origin, then a zero-energy resonance is present. A zero-energy resonance 
and a singularity cannot occur simultaneously. Let us attach to each function $F(k)$ an index $\alpha=-1,0,1$, which is equal to -1 in the case of a zero-energy singularity, 1 in the case of a zero-energy resonance and 0 otherwise. The following definition will be used in the future:

Definition 2. The set $\mathcal{F}$ is the set of functions $F(k)$ which can be presented in the following form

$$
F(k)=\frac{k+\mathrm{i} a_{0}}{k}\left(\prod_{j=1}^{N_{\text {sing }}} \frac{k+\mathrm{i} a_{j}}{k-b_{j}} \frac{k+\mathrm{i} \bar{a}_{j}}{k+b_{j}}\right) F_{F}(k)
$$

where function $F_{F}(k)$ is a Jost function from the Faddeev class $\mathcal{F}_{F}, a_{0} \geqslant 0, \operatorname{Re} a_{j}>0$. If $F_{F}(0)=0$, then $a_{0}=0$.

The absolute value of the Jost function defines the spectral density of the Schrödinger operator $\rho(k)=(2 / \pi)\left(k^{2} /|F(k)|^{2}\right)$. The spectral density, corresponding to the Jost functions from $\mathcal{F}$, vanishes at the points $\pm b_{j}$. The spectral measure can be calculated from the energies of the bound states, corresponding normalizing constants and the spectral density on the positive half-axis. The standard set of scattering data includes the scattering matrix, energies of the bound states and the normalizing constants. For potentials from the Faddeev class this information is enough to reconstruct the spectral measure. But zeros of the spectral density, corresponding to the Jost functions from $\mathcal{F}$, cannot be reconstructed from these scattering data. That is why we are going to consider the following set of scattering data:

Definition 3. The set $\mathcal{D}$ of scattering data consists of the following data:

(1) scattering matrix $S(k)$ from $\mathcal{S}$;

(2) natural numbers $N_{b s}, N_{\text {sing }} \in \mathbb{N}$ and $\alpha=-1,0,1$ such that

$$
\left.\arg S(k)\right|_{-\infty} ^{\infty}=-2 \pi\left(2 N_{b s}-2 N_{\text {sing }}+\alpha\right)
$$

(3) the energies of the bound states and corresponding normalizing constants

$$
E_{j}<0 \quad s_{j}>0 \quad j=1,2, \ldots, N_{b s}
$$

(4) positions of the zeros of the spectral density on the real line

$$
b_{j}>0 \quad j=1,2, \ldots, N_{\text {sing. }} .
$$

Lemma 1. Let $F(k)$ be any function from $\mathcal{F}$, then the quotient

$$
S(k)=\frac{F(-k)}{F(k)}
$$

is a function from $\mathcal{S}$. Let $S(k)$ be any function from $\mathcal{S}$, then there exists $F(k) \in \mathcal{F}$, such that (5) holds. The function $F(k)$ from $\mathcal{F}$ is defined uniquely by the scattering data from $\mathcal{D}$.

Proof. Suppose that $F(k) \in \mathcal{F}$, then representation (4) gives the following equality

$$
S(k)=\frac{F(-k)}{F(k)}=\frac{k-\mathrm{i} a_{0}}{k+\mathrm{i} a_{0}}\left(\prod_{j=1}^{N_{\text {sing }}} \frac{k-\mathrm{i} a_{j}}{k+\mathrm{i} a_{j}} \frac{k-\mathrm{i} \bar{a}_{j}}{k+\mathrm{i} \bar{a}_{j}}\right) \frac{F_{F}(-k)}{F_{F}(k)} .
$$

It follows that $S(k) \in \mathcal{S}$ because $S_{F}(k)=F_{F}(-k) / F_{F}(k) \in \mathcal{S}_{F}$.

Let function $S(k)$ be a function from $\mathcal{S}$ and the necessary data (2)-(4) are given. Let us consider the function

$$
\hat{S}(k)=\frac{k-\mathrm{i} c}{k+\mathrm{i} c} \frac{k+\mathrm{i} a_{0}}{k-\mathrm{i} a_{0}}\left(\prod_{j=1}^{N_{\text {sing }}} \frac{k+\mathrm{i} a_{j}}{k-\mathrm{i} a_{j}} \frac{k+\mathrm{i} \bar{a}_{j}}{k-\mathrm{i} \bar{a}_{j}}\right)\left(\prod_{j=1}^{N_{b s}} \frac{\left(k-\mathrm{i} \gamma_{j}\right)^{2}}{\left(k+\mathrm{i} \gamma_{j}\right)^{2}}\right) S(k)
$$


defined by certain constants $a_{0}, c \geqslant 0, \operatorname{Re} a_{j}>0, \gamma_{j}=\sqrt{-E_{j}}$ such that

$$
\begin{array}{rlrl}
\alpha & =-1 \rightarrow a_{0} \neq 0 & & c=0 \\
\alpha & =0 \rightarrow a_{0}=0 & c & =0 \\
\alpha & =1 \rightarrow a_{0}=0 & c \neq 0 .
\end{array}
$$

The function $\hat{S}(k)$ is a scattering matrix with zero phase shift on the real axis and the corresponding Jost function can be reconstructed in a unique way [11]. The phase of the scattering matrix can be defined by the following conditions

$$
\hat{\delta}(k)=\frac{\mathrm{i}}{2} \ln \hat{S}(k) \quad \hat{\delta}(0)=0 .
$$

Each such function $\hat{\delta}(k)$ is a sine-transformation of a certain function $\gamma \in L_{1}(0, \infty)$

$$
\hat{\delta}(k)=-\int_{0}^{\infty} \gamma(t) \sin k t \mathrm{~d} t \quad \int_{0}^{\infty}|\gamma(t)| \mathrm{d} t<\infty .
$$

Then the corresponding Jost function is equal to

$$
\hat{F}(k)=\exp \left(\int_{0}^{\infty} \gamma(t) \exp (\mathrm{i} k t) \mathrm{d} t\right) .
$$

The Jost function, which solves the original problem, is equal to

$$
F(k)=\frac{k}{k+\mathrm{i} c} \frac{k+\mathrm{i} a_{0}}{k}\left(\prod_{j=1}^{N_{\text {sing }}} \frac{k+\mathrm{i} a_{j}}{k-b_{j}} \frac{k+\mathrm{i} \bar{a}_{j}}{k+b_{j}}\right)\left(\prod_{n=1}^{N_{b s}} \frac{k-\mathrm{i} \gamma_{n}}{k+\mathrm{i} \gamma_{n}}\right) \tilde{F}(k) .
$$

Making different choices for the constants $a_{0}, c, a_{j}$ does not change the final function. The lemma is proven.

We have proven a slightly more general result. Let us use the following definition:

Definition 4. The set of spectral measures $\mathcal{M}$ is the set of all measures on the real line, which are pure point on the negative half-axis having there a finite number of atoms and pure continuous on the positive half-axis with the density function $\rho(k)=$ $(2 / \pi)\left(k^{2} /|F(k)|^{2}\right), F(k) \in \mathcal{F}$.

The following lemma has been proven:

Lemma 2. The scattering data from $\mathcal{D}$ and the spectral measures from $\mathcal{M}$ are in one-to-one correspondence.

\section{The inverse problem}

We are going to prove that for any scattering data from $\mathcal{D}$ there exists a certain potential $V_{S}(x)$ from $L_{2}(0, \infty)$ such that the Schrödinger operator $H=-\frac{\mathrm{d}^{2}}{\mathrm{~d} x^{2}}+V_{S}$ defines these scattering data. A similar problem has been studied for potentials from Faddeev's class of potentials with a finite first momentum $\mathcal{V}_{F}=\left\{V: \int_{0}^{\infty}(1+x)|V(x)| \mathrm{d} x<\infty\right\}$. We are going to extend this class in order to include potentials with long-range oscillating tails at infinity. The following class of potentials will be considered:

Definition 5. The set $\mathcal{V}$ of potentials is the set of all locally integrable potentials $V(x)$ on the half-axis having the following representation

$$
V(x)=\frac{\sum_{j=1}^{N} c_{j} \sin 2\left(b_{j} x+\beta_{j}\right)}{x+1}+V_{2}(x)+V_{F}(x)
$$

where $V_{2}(x)=\mathrm{O}\left(1 /(1+x)^{2}\right) ; \int_{0}^{\infty}(1+x)\left|V_{F}\right| \mathrm{d} x<\infty$ and constants $c_{j}, b_{j}, \beta_{j}$ are real. 
It is obvious that the set $\mathcal{V}$ includes the set of potentials with a finite first momentum $\mathcal{V}_{F} \subset \mathcal{V}$. A standard scattering theory can be developed for potentials from $\mathcal{V}$ [19, 27]. The usual Jost solution exists for all real values of $k \neq 0, k \neq b_{j}$ [4]. The Jost function is continuous on every closed interval outside these points. To solve the inverse problem for the scattering data from $\mathcal{D}$ it is enough to consider only Jost functions having simple poles at the real points $b_{j}$ and analytical in the upper half-plane $\operatorname{Im} k>0$. Let us denote the corresponding subset of potentials by $\mathcal{V}_{C}$.

Definition 6. The subset $\mathcal{V}_{C} \subset \mathcal{V}$ is the set of all potentials having a Jost function from $\mathcal{F}$.

Every scattering matrix $S(k)$ with the finite phase shift on the real axis can be presented in the form (3). Thus let us investigate the solution of the inverse scattering problem for the rational scattering matrices with a positive phase shift on the real axis. The phase shift is proportional to $2 \pi$, i.e. there exists a natural number $M$ such that $\left.\arg S(k)\right|_{-\infty} ^{+\infty}=M 2 \pi$. We have to separate out two possible cases: when the constant $M$ is an even or an odd number. In the second case the Jost function corresponding to the scattering matrix has a singularity at the origin. The following two lemmas consider the cases when the constant $M$ is even or equal to 1 .

Lemma 3. Let the potential $\tilde{V} \in \mathcal{V}_{F}$ and function

$$
S_{1}(k)=\prod_{j=1}^{N_{\text {sing }}} \frac{k-\mathrm{i} a_{j}}{k+\mathrm{i} a_{j}} \frac{k-\mathrm{i} \bar{a}_{j}}{k+\mathrm{i} \bar{a}_{j}}
$$

be given, then there exists a potential $V_{1}(x) \in \mathcal{V}$ such that $S_{1}(k)$ is a scattering matrix for the pair of operators

$$
\tilde{H}=-\frac{\mathrm{d}^{2}}{\mathrm{~d} x^{2}}+\tilde{V} \quad H_{1}=-\frac{\mathrm{d}^{2}}{\mathrm{~d} x^{2}}+\tilde{V}+V_{1} .
$$

Proof. The function $S_{1}(k)$ is not a scattering matrix from Faddeev's class, thus let us consider approximating the scattering matrix $S_{\epsilon} \in \mathcal{S}_{F}$ for arbitrary positive $\epsilon>0$

$$
S_{\epsilon}(k)=\prod_{j=1}^{N_{\text {sing }}} \frac{k-\mathrm{i} a_{j}}{k+b_{j}-\mathrm{i} \epsilon} \frac{k-\mathrm{i} \bar{a}_{j}}{k-b_{j}-\mathrm{i} \epsilon} \frac{k+b_{j}+\mathrm{i} \epsilon}{k+\mathrm{i} a_{j}} \frac{k-b_{j}+\mathrm{i} \epsilon}{k+\mathrm{i} \bar{a}_{j}} .
$$

Real numbers $b_{j}, j=1,2, \ldots, N_{b s}$ can be chosen arbitrarily. The inverse problem can be solved for $S_{\epsilon}$ in the class $\mathcal{V}_{F}$ of potentials. The corresponding Jost function is continuous in the closed upper half-plane

$$
F_{\epsilon}(k)=\prod_{j=1}^{N_{\text {sing }}} \frac{k+\mathrm{i} a_{j}}{k-b_{j}+\mathrm{i} \epsilon} \frac{k+\mathrm{i} \bar{a}_{j}}{k+b_{j}+\mathrm{i} \epsilon} .
$$

Let us denote by $f(k, x)$ and $\varphi(k, x)$ the Jost and regular solutions of the differential equation

$$
-\frac{\mathrm{d}^{2} y(k, x)}{\mathrm{d} x^{2}}+\tilde{V}(x) y(k, x)=k^{2} y(k, x) .
$$

Then the potential $V_{\epsilon}$ which solves the problem, is equal to

$$
V_{\epsilon}(x)=-2 \frac{\partial^{2}}{\partial x^{2}} \log \operatorname{det} \mathbf{W}(x, \epsilon) .
$$


Matrix $\mathbf{W}(x, \epsilon)$ has dimension $2 N_{\text {sing }} \times 2 N_{\text {sing }}$ with the following elements

$$
\begin{aligned}
& W_{2 l-1,2 j-1}(x, \epsilon)=\frac{1}{a_{l}^{2}+\left(b_{j}-\mathrm{i} \epsilon\right)^{2}} W\left[f\left(\mathrm{i} a_{l}, x\right), \varphi\left(-b_{j}+\mathrm{i} \epsilon, x\right)\right] \\
& W_{2 l, 2 j-1}(x, \epsilon)=\frac{1}{\bar{a}_{l}^{2}+\left(b_{j}-\mathrm{i} \epsilon\right)^{2}} W\left[f\left(\mathrm{i} \bar{a}_{l}, x\right), \varphi\left(-b_{j}+\mathrm{i} \epsilon, x\right)\right] \\
& W_{2 l-1,2 j}(x, \epsilon)=\frac{1}{a_{l}^{2}+\left(b_{j}+\mathrm{i} \epsilon\right)^{2}} W\left[f\left(\mathrm{i}_{l}, x\right), \varphi\left(b_{j}+\mathrm{i} \epsilon, x\right)\right] \\
& W_{2 l, 2 j}(x, \epsilon)=\frac{1}{\bar{a}_{l}^{2}+\left(b_{j}+\mathrm{i} \epsilon\right)^{2}} W\left[f\left(\mathrm{i} \bar{a}_{l}, x\right), \varphi\left(b_{j}+\mathrm{i} \epsilon, x\right)\right] \\
& j=1,2, \ldots, N_{b s} \quad l=1,2, \ldots, N_{b s} .
\end{aligned}
$$

$W[*, *]$ denotes here the Wronskian of two solutions of the differential equation. The regular solution is given by the formula

$$
\varphi_{\epsilon}(k, x)=\frac{\operatorname{det}\left|\begin{array}{cc}
\mathbf{W}(x) & f(x) \\
\beta(k, x) & \varphi(k, x)
\end{array}\right|}{\operatorname{det} \mathbf{W}(x)} .
$$

Vectors

$$
\begin{aligned}
& (f(x))_{2 l-1}=f\left(\mathrm{i} a_{l}, x\right) \quad(f(x))_{2 l}=f\left(\mathrm{i} \bar{a}_{l}, x\right) \\
& \beta_{2 j-1}=\int_{0}^{x} \varphi\left(-b_{j}+\mathrm{i} \epsilon, t\right) \varphi(k, t) \mathrm{d} t \quad \beta_{2 j}=\int_{0}^{x} \varphi\left(b_{j}+\mathrm{i} \epsilon, t\right) \varphi(k, t) \mathrm{d} t
\end{aligned}
$$

have dimension $2 N_{b s}$. Let us now consider the limit $\epsilon \rightarrow 0$ of the potential and regular solution. The determinant of the matrix $\mathbf{W}(x, 0)$ is equal to zero and the following representation holds

$$
\operatorname{det} \mathbf{W}(x, \epsilon)=(2 \epsilon)^{N_{b s}} \operatorname{det} \mathbf{Q}(x)+o\left((\epsilon)^{N_{b s}}\right)
$$

where $\mathbf{Q}(x)$ is a $2 N_{\text {sing }} \times 2 N_{\text {sing }}$ matrix with the following elements:

$$
\begin{aligned}
& Q_{2 l-1,2 j-1}(x)=\frac{2 b_{j} \mathrm{i}}{\left(a_{l}^{2}+b_{j}^{2}\right)^{2}} W\left[f\left(\mathrm{i} a_{l}, x\right), \varphi\left(b_{j}, x\right)\right]-\frac{\mathrm{i}}{a_{l}^{2}+b_{j}^{2}} W\left[f\left(\mathrm{i} a_{l}, x\right), \frac{\partial \varphi}{\partial k}\left(b_{j}, x\right)\right] \\
& Q_{2 l-1,2 j}(x)=\frac{1}{a_{l}^{2}+b_{j}^{2}} W\left[f\left(\mathrm{i} a_{l}, x\right), \varphi\left(b_{j}, x\right)\right] \\
& Q_{2 l, 2 j-1}(x)=-\overline{Q_{2 l-1,2 j-1}(x)} \quad Q_{2 l, 2 j}(x)=\overline{Q_{2 l, 2 j-1}(x)} \\
& j=1,2, \ldots, N_{b s} \quad l=1,2, \ldots, N_{b s} .
\end{aligned}
$$

We are going to prove first, that the determinant of the matrix $\mathbf{Q}$ is not equal to zero. If it is equal to zero at a certain point on the positive part of the real axis, then the limit potential has an inverse square singularity at this point and the corresponding potential is not locally integrable. The inverse problem under investigation can be solved by steps introducing singularities of the Jost function on the real line by pairs. In this way we obtain a representation of the limit potential as a sum of potentials obtained at each step. We are going to show that the determinants obtained at each step are not equal to zero. It is enough to consider the case $N_{\text {sing }}=1$. Direct calculations show in this case that

$$
\begin{aligned}
\operatorname{det} \mathbf{Q}(x)= & \frac{4 b \mathrm{i}\left(\bar{a}^{2}-a^{2}\right)}{\left(a^{2}+b^{2}\right)\left(\bar{a}^{2}+b^{2}\right)}\left\{\frac{W[f(\mathrm{i} a, x), \varphi(b, x)]}{a^{2}+b^{2}} \frac{W[f(\mathrm{i} \bar{a}, x), \varphi(b, x)]}{\bar{a}^{2}+b^{2}}\right. \\
& \left.+\frac{W[\varphi(b, x), \partial \varphi(b, x) / \partial k]}{2 b} \frac{W[f(\mathrm{i} a, x), f(\mathrm{i} \bar{a}, x)]}{\bar{a}^{2}-a^{2}}\right\} .
\end{aligned}
$$


The last formula can be simplified using the following properties of the Wronskians, which are valid for any locally integrable potential. Let $y(k, x)$ be any solution of the Schrödinger equation (6) corresponding to the energy $k^{2}$, then the following equality holds

$$
\left.W\left[y(k, x), y\left(k^{\prime}, x\right)\right]\right|_{x_{1}} ^{x_{2}}=\left(k^{2}-k^{\prime 2}\right) \int_{x_{1}}^{x_{2}} y(k, x) y\left(k^{\prime}, x\right) \mathrm{d} x .
$$

We have, in particular, for the regular solution

$$
W\left[\varphi(b, x), \frac{\partial \varphi}{\partial k}(b, x)\right]=-2 b \int_{0}^{x} \varphi^{2}(b, t) \mathrm{d} t .
$$

The following representation for the determinant is valid

$$
\begin{aligned}
\operatorname{det} \mathbf{Q}(x)= & \frac{4 b \mathrm{i}\left(\bar{a}^{2}-a^{2}\right)}{\left(a^{2}+b^{2}\right)\left(\bar{a}^{2}+b^{2}\right)}\left\{\int_{x}^{\infty} f(\mathrm{i} a, t) \varphi(b, t) \mathrm{d} t \int_{x}^{\infty} \bar{f}(\mathrm{i} a, t) \varphi(b, t) \mathrm{d} t\right. \\
& \left.+\int_{0}^{x} \varphi^{2}(b, t) \mathrm{d} t \int_{x}^{\infty}|f(\mathrm{i} a, t)|^{2} \mathrm{~d} t\right\} .
\end{aligned}
$$

We have used here the fact that $f(\mathrm{i} \bar{a}, x)=\bar{f}(\mathrm{i} a, x)$. Formula (7) shows that the determinant of $\mathbf{Q}$ is not equal to zero, because the expression in the curly brackets is always positive for positive $x: x>0 \Rightarrow \operatorname{det} \mathbf{Q}(x) \neq 0$. We conclude that the limit potential is given by the usual formula

$$
V_{1}(x)=-2 \frac{\partial^{2}}{\partial x^{2}} \log \operatorname{det} \mathbf{Q}(x)=-2 \frac{(\operatorname{det} \mathbf{Q})^{\prime \prime} \operatorname{det} \mathbf{Q}-\left(\operatorname{det} \mathbf{Q}^{\prime}\right)^{2}}{(\operatorname{det} \mathbf{Q})^{2}} .
$$

This formula is valid for an arbitrary number of singularities $N_{\text {sing }}$. Let us study the asymptotics of this potential. We substitute the asymptotics of the regular and Jost solutions

$$
\begin{aligned}
& \varphi\left(b_{j}, x\right) \sim_{x \rightarrow \infty} A\left(b_{j}\right) \sin \left(b_{j} x+\delta\left(b_{j}\right)\right) \\
& f\left(\mathrm{i} a_{l}, x\right) \sim_{x \rightarrow \infty} \mathrm{e}^{-a_{l} x}
\end{aligned}
$$

into the matrix $\mathbf{Q}$

$$
\begin{aligned}
Q_{2 l-1,2 j-1}^{a s}(x)= & \frac{2 b_{j} \mathrm{i}}{\left(a_{l}^{2}+b_{j}^{2}\right)^{2}} A\left(b_{j}\right) \mathrm{e}^{-a_{l} x}\left(b_{j} \cos \left(b_{j} x+\delta\left(b_{j}\right)\right)+a_{l} \sin \left(b_{j} x+\delta\left(b_{j}\right)\right)\right) \\
& -\frac{\mathrm{i}}{a_{l}^{2}+b_{j}^{2}} \mathrm{e}^{-a_{l} x}\left(\cos \left(b_{j} x+\delta\left(b_{j}\right)\right)\left(A^{\prime}\left(b_{j}\right) b_{j}+A\left(b_{j}\right)+A\left(b_{j}\right) a_{l}\left(x+\delta^{\prime}\left(b_{j}\right)\right)\right)\right. \\
& \left.+\sin \left(b_{j} x+\delta\left(b_{j}\right)\right)\left(-A\left(b_{j}\right) b_{j}\left(x+\delta^{\prime}\left(b_{j}\right)\right)+A^{\prime}\left(b_{j}\right) a_{l}\right)\right) \\
Q_{2 l-1,2 j}^{a s}(x)= & \frac{A\left(b_{j}\right) \mathrm{e}^{-a_{l} x}}{a_{l}^{2}+b_{j}^{2}}\left(b_{j} \cos \left(b_{j} x+\delta\left(b_{j}\right)\right)+a_{l} \sin \left(b_{j} x+\delta\left(b_{j}\right)\right)\right) \\
Q_{2 l, 2 j-1}^{a s}(x)= & -\overline{Q_{2 l-1,2 j-1}^{a s}(x)} \quad Q_{2 l, 2 j}^{a s}(x)=\overline{Q_{2 l, 2 j-1}^{a s}(x)} .
\end{aligned}
$$

The terms containing $A^{\prime}\left(b_{j}\right)$ do not give any contribution to the determinants. The determinant of the matrix $\mathbf{Q}$ divided by $\exp \left(-2 \sum_{l=1}^{N_{\text {sing }}} \operatorname{Re} a_{l} x\right)$ is a polynomial with respect to $x$ of order $N_{\text {sing }}$ with the coefficients equal to certain trigonometric functions:

$\operatorname{det} \mathbf{Q}(x)=\exp \left(-2 \sum_{l=1}^{N_{\text {sing }}} \operatorname{Re} a_{l} x\right)\left(p_{0}(x) x^{N_{\text {sing }}}+p_{1}(x) x^{N_{\text {sing }}-1}+\cdots+p_{N_{\text {sing }}}(x)\right)$.

Multiplication by the exponential function $\exp \left(-2 \sum_{l=1}^{N_{\text {sing }}} \operatorname{Re} a_{l} x\right)$ gives a zero contribution to the formula for potential (8) after taking the second derivative of the logarithm. The long-range oscillating asymptotics of the potential can be calculated using the first two 
functions only: $p_{0}(x)$ and $p_{1}(x)$. We are going to prove that the function $p_{0}(x)$ is equal to a constant. This function is equal to the determinant of the matrix $P^{0}$ with the following coefficients:

$$
\begin{aligned}
& P_{2 l-1,2 j-1}^{0}(x)=-\frac{\mathrm{i}}{a_{l}^{2}+b_{j}^{2}} A\left(b_{j}\right)\left(a_{l} \cos \left(b_{j} x+\delta\left(b_{j}\right)\right)-b_{j} \sin \left(b_{j} x+\delta\left(b_{j}\right)\right)\right) \\
& P_{2 l-1,2 j}^{0}(x)=\frac{A\left(b_{j}\right)}{a_{l}^{2}+b_{j}^{2}}\left(b_{j} \cos \left(b_{j} x+\delta\left(b_{j}\right)\right)+a_{l} \sin \left(b_{j} x+\delta\left(b_{j}\right)\right)\right) \\
& P_{2 l, 2 j-1}^{0}(x)=-\overline{P_{2 l-1,2 j-1}^{0}(x)} \quad P_{2 l, 2 j}^{0}(x)=\overline{P_{2 l, 2 j-1}^{0}(x)} .
\end{aligned}
$$

The derivative of $p_{0}(x)$ is equal to the sum of the determinants of the matrices, which can be obtained from the matrix $P^{0}$ by substituting the $n$th column by its derivative with respect to $x$. Each odd column of the matrix $P^{0}$ is equal to the derivative of the next even column divided by $\mathrm{i} b_{j}$. Every element of the $(2 j-1)$ th and $2 j$ th columns is a solution of the equation $-y^{\prime \prime}(x)=b_{j}^{2} y(x)$. It follows that each matrix used for the calculation of the derivative of $p_{0}$ has two linearly dependent columns and the corresponding determinants are equal to zero. We have proven that the derivative of $p_{0}$ is equal to zero. One can show that the determinant of the matrix $P^{0}$ does not vanish [17].

Let us investigate the function $p_{1}(x)$. It is equal to the sum of the determinants of $N_{\text {sing }}$ matrices $P^{1, j}$, which can be obtained from the matrix $P^{0}$ by substituting the $(2 j-1)$ th column with the following one:

$$
\begin{aligned}
P_{2 l-1,2 j-1}^{1, j}(x)= & \frac{2 b_{j} \mathrm{i}}{\left(a_{l}^{2}+b_{j}^{2}\right)^{2}} A\left(b_{j}\right)\left(b_{j} \cos \left(b_{j} x+\delta\left(b_{j}\right)\right)+a_{l} \sin \left(b_{j} x+\delta\left(b_{j}\right)\right)\right) \\
& -\frac{\mathrm{i}}{a_{l}^{2}+b_{j}^{2}}\left(\cos \left(b_{j} x+\delta\left(b_{j}\right)\right)\left(A\left(b_{j}\right)+A\left(b_{j}\right) a_{l} \delta^{\prime}\left(b_{j}\right)\right)\right. \\
& \left.-\sin \left(b_{j} x+\delta\left(b_{j}\right)\right) A\left(b_{j}\right) b_{j} \delta^{\prime}\left(b_{j}\right)\right) .
\end{aligned}
$$

We first separate out the contribution due to the phases $\delta^{\prime}\left(b_{j}\right)$ :

$$
p_{1}(x)=p_{0} \sum_{j=1}^{N_{b s}} \delta^{\prime}\left(b_{j}\right)+\sum_{j=1}^{N_{\text {sing }}} \operatorname{det} \hat{P}^{1, j}
$$

where matrices $\hat{P}^{1, j}$ have the following $(2 j-1)$ th columns

$$
\begin{aligned}
\hat{P}_{2 l-1,2 j-1}^{1, j}(x)= & \frac{2 b_{j} \mathrm{i}}{\left(a_{l}^{2}+b_{j}^{2}\right)^{2}} A\left(b_{j}\right)\left(b_{j} \cos \left(b_{j} x+\delta\left(b_{j}\right)\right)+a_{l} \sin \left(b_{j} x+\delta\left(b_{j}\right)\right)\right) \\
& -\frac{\mathrm{i}}{a_{l}^{2}+b_{j}^{2}} \cos \left(b_{j} x+\delta\left(b_{j}\right)\right) A\left(b_{j}\right)
\end{aligned}
$$

and the same other columns. The determinants of these matrices can be presented in the following form

$$
\operatorname{det} \hat{P}^{1, j}=\sum_{b} c_{j}(b) \sin 2\left(b x+\beta_{j}(b)\right)
$$

where constants $b$ belong to the set of all sums and differences of the constants $b_{j}$ : $B\left(\left\{b_{j}\right\}_{j=1}^{N}\right)=\left\{b: b=\sum_{j=1}^{N} \alpha_{j} b_{j}, \alpha_{j}= \pm 1\right\}$. The sum of all these determinants have a similar structure:

$$
p_{1}(x)=\sum_{b \neq 0} c(b) \sin 2(b x+\beta(b))+c_{0} .
$$


Then the asymptotics of the potential is equal to

$$
V(x) \sim_{x \rightarrow \infty}-2 \frac{p_{1}^{\prime \prime}(x)}{p_{0}(x+1)}+\mathrm{O}\left(\frac{1}{(x+1)^{2}}\right)
$$

and it follows that potential $V_{1}$ belongs to the set $\mathcal{V}$.

The limit of the regular solution is given by the formula

$$
\varphi_{1}(k, x)=\frac{\operatorname{det}\left|\begin{array}{cc}
\mathbf{Q}(x) & f(x) \\
\beta_{1}(k, x) & \varphi(k, x)
\end{array}\right|}{\operatorname{det} \mathbf{Q}(x)}
$$

with the following vectors

$\beta_{1,2 j-1}=-\mathrm{i} \int_{0}^{x}\left(\frac{\partial}{\partial k} \varphi\left(b_{j}, t\right)\right) \varphi(k, t) \mathrm{d} t \quad \beta_{1,2 j}=\int_{0}^{x} \varphi\left(b_{j}, t\right) \varphi(k, t) \mathrm{d} t$.

Formulae (8) and (9) represent Darboux transformations [20] for the Schrödinger equation (6). It follows that the $\varphi_{1}(k, x)$ given by (9) is a regular solution for the operator $H_{1}$ with $V_{1}$ given by (8). The asymptotics of the regular solution can be calculated:

$\varphi_{1}(k, x) \sim_{x \rightarrow \infty} \frac{-1}{2 \mathrm{i} k}\left\{F(-k) \mathrm{e}^{\mathrm{i} k x} \prod_{j=1}^{N_{b s}} \frac{\left(k-\mathrm{i} a_{j}\right)\left(k-\mathrm{i} \bar{a}_{j}\right)}{k^{2}-b_{j}^{2}}-F(k) \mathrm{e}^{-\mathrm{i} k x} \prod_{j=1}^{N_{b s}} \frac{\left(k+\mathrm{i} a_{j}\right)\left(k+\mathrm{i} \bar{a}_{j}\right)}{k^{2}-b_{j}^{2}}\right\}$

and the scattering matrix is equal to $S_{1}(k)$. Lemma 3 is proven.

Lemma 4. Let potential $V$ be from the set $\mathcal{V}_{C}$ such that the Jost function $F_{V}(k)$ does not vanish at the origin $F_{V}(0) \neq 0$ and the operator does not have a zero-energy bound state. Then for any positive $0<a_{0}<\sqrt{-E_{1}}$ ( $E_{1}$ is the energy of the highest bound state) there exists a potential $V_{0} \in \mathcal{V}_{C}$ such that function $S_{0}(k)=\left(k-\mathrm{i} a_{0}\right) /\left(k+\mathrm{i} a_{0}\right)$ is a scattering matrix for the pair of operators

$$
H_{1}=-\frac{\mathrm{d}^{2}}{\mathrm{~d} x^{2}}+V \quad H_{0}=-\frac{\mathrm{d}^{2}}{\mathrm{~d} x^{2}}+V+V_{0}
$$

Proof. One can apply an approximation procedure similar to the one used in the proof of lemma 3. The solution of the inverse problem is given by the following formula:

$V_{0}(x)=2 a^{2} \frac{f_{1}^{2}\left(\mathrm{i} a_{0}, x\right) \varphi_{1}^{\prime 2}(0, x)-f_{1}^{\prime 2}\left(\mathrm{i} a_{0}, x\right) \varphi_{1}^{2}(0, x)+a^{2} f_{1}^{2}\left(\mathrm{i} a_{0}, x\right) \varphi_{1}^{2}(0, x)}{\left(f_{1}\left(\mathrm{i} a_{0}, x\right) \varphi_{1}^{\prime}(0, x)-f_{1}^{\prime}\left(\mathrm{i} a_{0}, x\right) \varphi_{1}(0, x)\right)^{2}}$

where $\varphi_{1}(k, x), f_{1}(k, x)$ are the regular and Jost solutions for the Schrödinger operator $H_{1}$. We are going to prove that the denominator in the last formula does not vanish. Suppose that the Wronskian is equal to zero:

$$
W\left[f_{1}\left(\mathrm{i} a_{0}, x_{0}\right), \varphi_{1}\left(0, x_{0}\right)\right]=0
$$

at some point $x_{0}>0$. Let us consider two functions

$$
g(k)=\frac{f_{1}^{\prime}\left(\mathrm{i} k, x_{0}\right)}{f_{1}\left(\mathrm{i} k, x_{0}\right)} \quad h(k)=\frac{h_{1}^{\prime}\left(\mathrm{i} k, x_{0}\right)}{h_{1}\left(\mathrm{i} k, x_{0}\right)} .
$$

These functions are similar to the Weyl function for the differential operator [27]. Both functions are meromorphic functions of $k$ with possible singularities on the real axis. These functions are real for the real values of the variable $k$. Function $g(k)$ has a negative imaginary part in the upper half-plane $\operatorname{Im} k>0$, but $h(k)$ has a positive imaginary part there. It follows that $g(k)$ is a decreasing function on every interval on the positive halfaxis outside the singular points. Function $h(k)$ is an increasing function on every similar 
interval. Condition (10) means that $g\left(a_{0}\right)=h(0)$ and it follows that there exists a certain point $0<k_{0}<a_{0}$, such that $g\left(k_{0}\right)=h\left(k_{0}\right)$. However, the last equation means that the point $E=-k_{0}^{2}$ is a discrete spectrum point of the operator $H_{1}$, which contradicts the condition $a_{0}<\sqrt{-E_{1}}$. The case $g\left(k_{0}\right)=h\left(k_{0}\right)=\infty$ means that the eigenfunction with the energy $-k_{0}^{2}$ is equal to zero at $x_{0}$. We have proven that the denominator does not vanish on the positive half-axis.

If potential $V \in \mathcal{V}_{C}$, then any solution of the Schrödinger equation for the zero energy is equivalent to a linear function in the limit $x \rightarrow \infty$. If the Jost function does not vanish at the origin, then the coefficient $\alpha$ in the asymptotics of the regular solution

$$
\varphi_{1}(0, x) \sim_{x \rightarrow \infty} \alpha x+\beta+o(1)
$$

is not equal to zero. The following asymptotics is valid for the derivative of the regular solution $\varphi_{1}^{\prime}(0, x) \sim_{x \rightarrow \infty} \alpha+o(1)$. The asymptotics of the potential is equal to

$$
V_{0}(x) \sim_{x \rightarrow \infty} \frac{2}{(1+x)^{2}}
$$

and it follows that $V_{0} \in \mathcal{V}$. The scattering matrix can be calculated using the regular solution

$\varphi_{0}(k, x)=\varphi_{1}(k, x)+\frac{a_{0}^{2}}{k^{2}} \frac{W\left[\varphi_{1}(0, x), \varphi_{1}(k, x)\right]}{W\left[\varphi_{1}(0, x), f_{1}\left(\mathrm{i} a_{0}, x\right)\right]} f_{1}\left(\mathrm{i} a_{0}, x\right)$

which has the following asymptotics

$\varphi_{0}(k, x) \sim_{x \rightarrow \infty} \frac{-1}{2 \mathrm{i} k}\left\{F_{1}(-k) \mathrm{e}^{\mathrm{i} k x} \frac{k-\mathrm{i} a_{0}}{k}-F_{1}(k) \mathrm{e}^{-\mathrm{i} k x} \frac{k+\mathrm{i} a_{0}}{k}\right\} \Rightarrow S_{0}(k)=\frac{k-\mathrm{i} a_{0}}{k+\mathrm{i} a_{0}}$.

Lemma 4 is proven.

It is possible to prove that the calculated potentials define no positive eigenvalues (see [17]). We are ready now to prove our main theorem.

Theorem 1. Let the scattering data

$$
S(k) ; N_{b s}, N_{\text {sing }}, \alpha ; E_{j}, s_{j}, j=1,2, \ldots, N_{b s} ; b_{j}, j=1,2, \ldots, N_{\text {sing }}
$$

from $\mathcal{D}$ be given, then there exists a potential $V \in \mathcal{V}$ corresponding to these scattering data. This potential is defined uniquely in the class $\mathcal{V}_{C}$.

Proof. This theorem can be proven using the Gelfand-Levitan-Marchenko procedure. We have shown that the scattering data from $\mathcal{D}$ define the spectral measure in a unique way. It follows that the potential from $\mathcal{V}_{C}$ is defined uniquely. It is enough to show that this potential exists and belongs to the class $\mathcal{V}$. This can be proven using the corresponding results for potentials with a finite first momentum. Any function $S(k) \in \mathcal{S}$ can be presented in the form (3). The constant $a_{0}$ in the representation can be chosen in such a way that $0<a_{0}<\sqrt{-E_{1}}$, where $E_{1}$ is the highest bound state of the operator.

The theorem can be proven in three steps. One can first construct the potential $V_{F} \in \mathcal{V}_{F}$ corresponding to the scattering matrix $S_{F}(k)$ and having $N_{b s}$ bound states with the energies $E_{j}$ and normalizing constants $s_{j}, j=1,2, \ldots, N_{b s}$. The solution of this problem is given, for example, in $[7,11]$. In the second step the inverse problem should be solved for the scattering matrix

$$
S_{1}(k)=\prod_{j=1}^{N_{\text {sing }}} \frac{k-\mathrm{i} a_{j}}{k+\mathrm{i} a_{j}} \frac{k-\mathrm{i} \bar{a}_{j}}{k+\mathrm{i} \bar{a}_{j}}
$$


considering potential $V_{F}$ as the background potential. The solution of this problempotential $V_{1} \in \mathcal{V}_{C}$-is given by lemma 3 . In the final step, if it is necessary, the zeroenergy singularity has to be introduced. We note that the constant $a_{0}$ is not equal to zero only if the function $S_{F}(k)$ is equal to one at the origin. Thus lemma 4 can be applied to find the potential $V \in \mathcal{V}_{C}$, which solves the inverse problem for the scattering matrix $S_{0}(k)=\left(k-\mathrm{i} a_{0}\right) /\left(k+\mathrm{i} a_{0}\right)$ with the background potential $V_{1}$. Theorem 1 is proven.

\section{Discussion}

Schrödinger operators with long-range oscillating potentials were first studied in relation to the bound states embedded in a continuous spectrum. Such eigenvalues can be easily considered in the framework of our approach. One can add a finite number of such eigenvalues using the closed form of the solution of the Gelfand-Levitan-Marchenko equation for the phase-equivalent potentials [7].

It is natural to generalize the discussed ideas to the scattering problem on the whole axis. A similar problem was studied in [26]. The authors extended the set of scattering matrices in order to include transition coefficients with zeros on the real axis. (The transition coefficient does not vanish there if the potential has a finite first momentum.) The solution of the inverse problem gives long-range oscillating potentials, depending on the positions of the zeros of the transition coefficient. However, no ambiguity appears in this case, because the zeros of the transition coefficient can be easily reconstructed from the reflection coefficient. Moreover, the potentials constructed in our case for $S_{F}(k)=1$ can be continued analytically for negative $x$. These potentials have second-order singularities on the negative half-axis. The scattering theory for such potentials can be defined using analytical continuation. It appears that the corresponding transition coefficient is identically equal to 1 and does not vanish on the real line. This generalized solution of the inverse problem has been used in the construction of the solutions of nonlinear evolution equations. The corresponding long-range solutions of the evolution equations were called positons [16,21-23]. We have to point out that the inverse scattering problem on the line cannot be solved uniquely even for potentials decaying at infinity like $\mathrm{O}\left(1 / x^{2}\right)$ (see $[2,3,8,24]$ and references therein). The corresponding ambiguity is related to the low-energy behaviour of the scattering matrix. The set of admissible potentials can be extended also in order to include distributions $[5,6]$. The first potentials without bound states having equal scattering matrices were constructed with the delta functional singularities. This shows another important extension of the class of admissible potentials.

\section{Acknowledgments}

The author wishes to thank Professor L Päivärinta for the invitation to a very stimulating conference in Saariselkä and Professors R Newton and K Chadan for the helpful suggestions. The author is indebted to all participants at the Mittag-Leffler seminar on mathematical physics in 1993-94 and especially to Professors M S Birman, V S Buslaev, P Deift and L A Pastur for constructive criticism. It is a pleasure for him to thank Professors J Boman, B S Pavlov and S N Naboko for continuous interest in the work. He is grateful to Professor $\mathrm{S}$ Albeverio for fruitful and encouraging discussions. Financial support by the Alexander von Humboldt foundation is gratefully acknowledged. 


\section{References}

[1] Albeverio S 1972 Ann. Phys., Lpz. 71 167-276

[2] Aktosun T and Newton R G 1985 Inverse Problems 1 291-300

[3] Aktosun T 1988 Inverse Problems 4 347-52

[4] Atkinson F 1954 Annali di Matematica Pura ed Applicata Serie Quatra, tomo XXXVII

[5] Atkinson D and Geschiere J 1986 Inverse Problems 2 L51-L53

[6] Atkinson D 1988 Meromorphic reflection coefficient Some Topics on Inverse Problems ed P C Sabatier (Singapore: World Scientific) pp 175-81

[7] Chadan K and Sabatier P C 1977 Inverse Problems in Quantum Scattering Theory 2nd edn (New York: Springer)

[8] Degasperis A and Sapatier P C 1987 Inverse Problems 3 73-109

[9] Deift P 1992 Differential Equations and Mathematical Physics (New York: Academic) pp 45-62

[10] Eastham M S P and Kalf H 1982 Schrödinger-type Operators with Continuous Spectra (London: Pitman)

[11] Faddeev L D 1963 J. Math. Phys. 4 72-104 (translated from 1959 Usp. Mat. Nauk 14 57)

[12] Gelfand I M and Levitan B M 1951 Izv. Akad. Nauk SSSR 15 309-60 (Engl. transl. 1955 Am. Math. Soc. Trans. Ser. 21 253-304)

[13] Gesztesy F and Simon B 1996 Uniqueness theorems in inverse spectral theory for one-dimensional Schrödinger operators Trans. Am. Math. Soc. 348 349-73

[14] Kurasov P B 1992 Lett. Math. Phys. 25 287-97

[15] Kurasov P B 1993 On the inverse scattering problem for rational reflection coefficients Inverse Problems in Mathematical Physics ed L Päivärinta and E Somersalo (Berlin: Springer) pp 126-33

[16] Kurasov P B 1993 On the soliton and positon solutions of the KdV equation Preprint MSI 93-1, ISSN-1100$214 \mathrm{X}$

[17] Kurasov P B 1995 Scattering matrices with finite phase shift and the inverse scattering problem Preprint ISSN: 1400-4003, ISRN: HLU-MAT-RES-95/14-SE

[18] Marchenko V A 1952 Trudy Moscov. Mat. Obsch. $1327-420$ (Engl. transl. 1973 Am. Math. Soc. Transl. Ser. 2101 1-104)

[19] Matveev V B and Skriganov M M 1972 Dokl. Acad. Nauk SSSR 202 755-8

[20] Matveev V B and Salle M A 1991 Darboux Transformations and Solitons (Berlin: Springer)

[21] Matveev V B 1992 Phys. Lett. 166A 205-8

[22] Matveev V B 1992 Phys. Lett. 166A 209-12

[23] Matveev V B 1994 J. Math. Phys. 35 2955-70

[24] Moses H E 1983 Phys. Rev. A 27 2220-1

[25] Newton R 1966 Scattering Theory for Waves and Particles (New York: McGraw-Hill)

[26] Novikov R G and Khenkin G M 1984 Teor. Mat. Fiz. 61 199-213

[27] Reed M and Simon B 1979 Methods of Modern Mathematical Physics, vol III Scattering Theory (New York: Academic) pp 155-68 